\title{
From Table-System to Tabletop: Integrating Technology into Interactive Surfaces
}

\section{Report}

Author(s):

Kunz, Andreas (1); Fjeld, Morten

Publication date:

2010

Permanent link:

https://doi.org/10.3929/ethz-a-006071005

Rights / license:

In Copyright - Non-Commercial Use Permitted 


\title{
From Table-System to Tabletop: Integrating Technology into Interactive Surfaces
}

\author{
Andreas Kunz ${ }^{1}$ and Morten Fjeld ${ }^{2}$ \\ ${ }^{1}$ Swiss Federal Institute of Technology, Department of Mechanical and Process Engineering \\ Tannenstrasse 3, CH-8092 Zurich, Switzerland \\ kunz@iwf.mavt.ethz.ch \\ ${ }^{2}$ Chalmers University of Technology, Dept. of Computer Science and Engineering, t2i Lab \\ Rännvägen 6b, SE-412 96 Gothenburg, Sweden \\ fjeld@chalmers.se
}

\begin{abstract}
Teamwork for generating new ideas is typically done on vertical or horizontal interaction spaces in order to capture volatile ideas by generating digital content that can be used during ongoing work. While many products are available for vertical interaction spaces, tabletop interaction is still subject to ongoing research and development. The following article gives a classification of existing tabletop systems according to the type of interaction, the means of tracking and identification, and the method of displaying images. Then, the challenges faced by researchers and the motivations behind their endeavours are discussed. We describe the evolution of this technology by citing important projects and their significance in the overall development of interactive tabletop surfaces. Finally, we give an outlook on future trends in this research field.
\end{abstract}

\section{Introduction and Classification}

The difference between a horizontal and a vertical interaction space is much more than an angle of $90^{\circ}$. The orientation has a significant impact on the user's behavior as well as on the employed technology.

Vertical interaction spaces utilize the metaphor of a whiteboard, and so, are typically designed as a single user interface. Their main purpose is to disseminate information, meaning that only one user with a single interaction device will work with the system per time interval. The most common interactions on these spaces are writing and sketching. In both cases, the user touches the surface with the pen's tip at one point at a time, which can be detected by various technologies. In addition, the interaction devices need only be detected and tracked for the duration of interaction, since they cannot remain on the interaction surface without any user 
interaction. Also, vertical interaction spaces have less challenging demands regarding the content orientation on the screen. Standard drop-down menus can be used, since all users will have the same perspective on the visible information.

Horizontal interactive spaces behave much differently. Tabletop systems are not primarily used to disseminate information, but to elaborate it by a single user or a small group gathered around the table. They are used to generate, manipulate, and display digital objects, which are carriers of information and thus the basis of discussion within a team. A horizontal surface also imposes new challenges in terms of system design. Within a typical tabletop interaction scenario, users stand or sit around the table and have very different views on the displayed content (see Figure 8 - Figure 12). So, typical interaction menus such as drop-down menus are not suitable since they are difficult to read from most positions around the table. Moreover, many devices are placed on the table that are partially meant for interaction. However, there are also devices on the table that are neither related to the system nor to the task at hand. From a technical point of view, this results in multiple points on the interactive surface that either need to be tracked and identified or ignored. In the act of writing, for example, users want to interact with the tip of the stylus, but they also touch the interactive surface with their hands and fingers. In this situation, the system should detect only the stylus and ignore the hand. However, in the next moment the user might use their fingers to move a virtual piece of paper around, which requires that the system now detects the fingers. Additionally, the stylus may be used for pointing onto certain objects. In this case, the system should detect the stylus even if it does not touch the tabletop's surface, but hovers a short distance above it.

During interaction on the table, the user frequently interferes with the light path that is required by most systems for image display and vision-based tracking. This results in shadow casting by the user or non-ergonomic working positions when sitting at the table. These challenges must be addressed within the fields of interaction, tracking and identification, image display, and software, which are described in more detail in the following paragraphs.

\section{Interaction}

Since a tabletop system is both a display and a direct input device, it must accept natural hand gestures and other intuitive devices as input [1]. This will improve the fluidity and reduce the cognitive load of the user-system interaction. Thus, the tabletop system must be able to distinguish between intended input using fingers and other devices, and unintended input by other objects on the table. Furthermore, tabletop systems must be able to detect multiple devices simultaneously, no matter if one or multiple users are interacting with the system. Figure 1 shows a classification of the types of interaction with a tabletop system. 


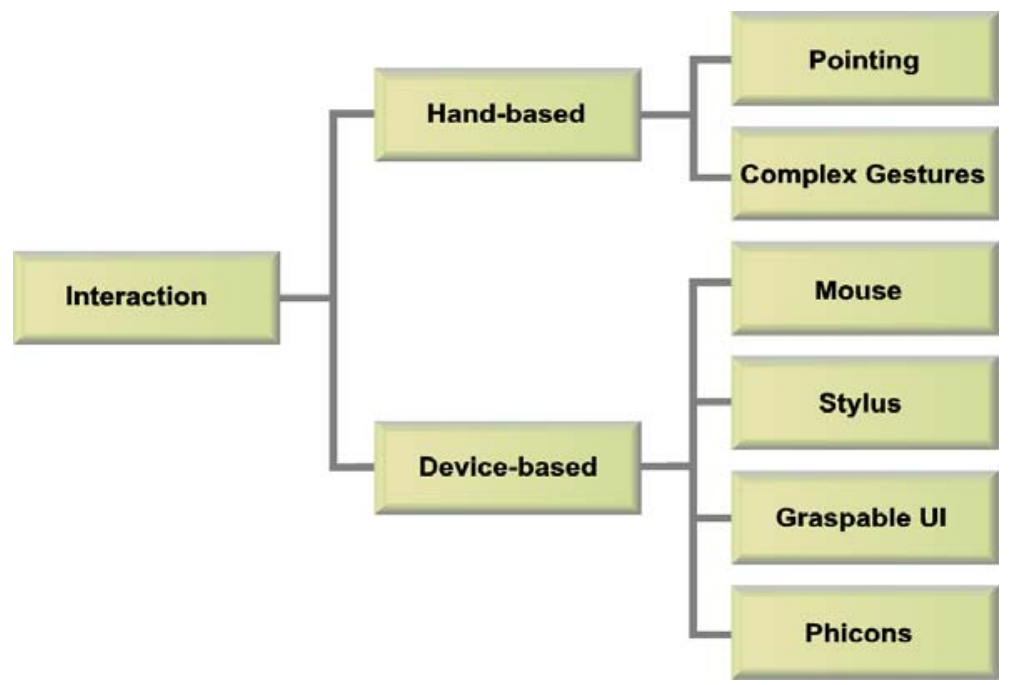

Figure 1. Classification of tabletop interaction

In order to enable intuitive interaction with the content that is visible on the tabletop, devices other than the mouse and keyboard must be used. There is a class of devices that are easily identifiable by their inherent function known as 'physical icons', or phicons [2]. In this case, each device usually has a static association so that the tabletop system is able to detect its identifier (ID) and not just its position. Once the device's ID is known to the system, the underlying functionality is also defined since the association cannot be changed. However, other tabletop systems have a dynamic association that allows for simpler detection algorithms. In the latter case, the devices have a more general character and so the intuitiveness is only guaranteed by the displayed content, i.e. the graphical user interface (GUI). The dynamic association is user-triggered and follows predefined steps. These steps may require some learning on the part of the user.

\section{Tracking and Identification}

Tabletop systems must be able to detect the position of an interaction device and, in case of phicons or other specialized input devices, their ID. While the position of a device is important for the interaction in a global context to be displayed on the tabletop's surface, the ID is relevant for integrating a device's specialized functionality into a specific application.

Although tracking has been well researched in the field of virtual reality, it is still quite a delicate task even on a 2D tabletop surface. More degrees of freedom (DOF) than given by planar interaction become relevant. For instance, the zcoordinate may be used to distinguish between writing and pointing in pen-based 
interaction. Additionally, the tracking and detection system's latency should be below the user's perceptual threshold, otherwise user irritation may occur.

An even more critical task for the tracking and identification system is distinguishing between objects meant for interaction, such as a finger, and objects not meant for interaction, such as coffee mugs or the side of a user's hand. During normal operation on a tabletop system, various objects could be placed on the surface which are not meant for interaction, but which could cause irritations to the tabletop system, e.g. by shadowing effects.

Unlike a mouse, which is a relative pointing device (i.e. the travelling distance and orientation are detected), all tracking systems for tabletop systems allow socalled absolute pointing, i.e. the object's detection is at the place where the user puts the device. Below, Figure 2 depicts the various types of tracking and detection that can be used.

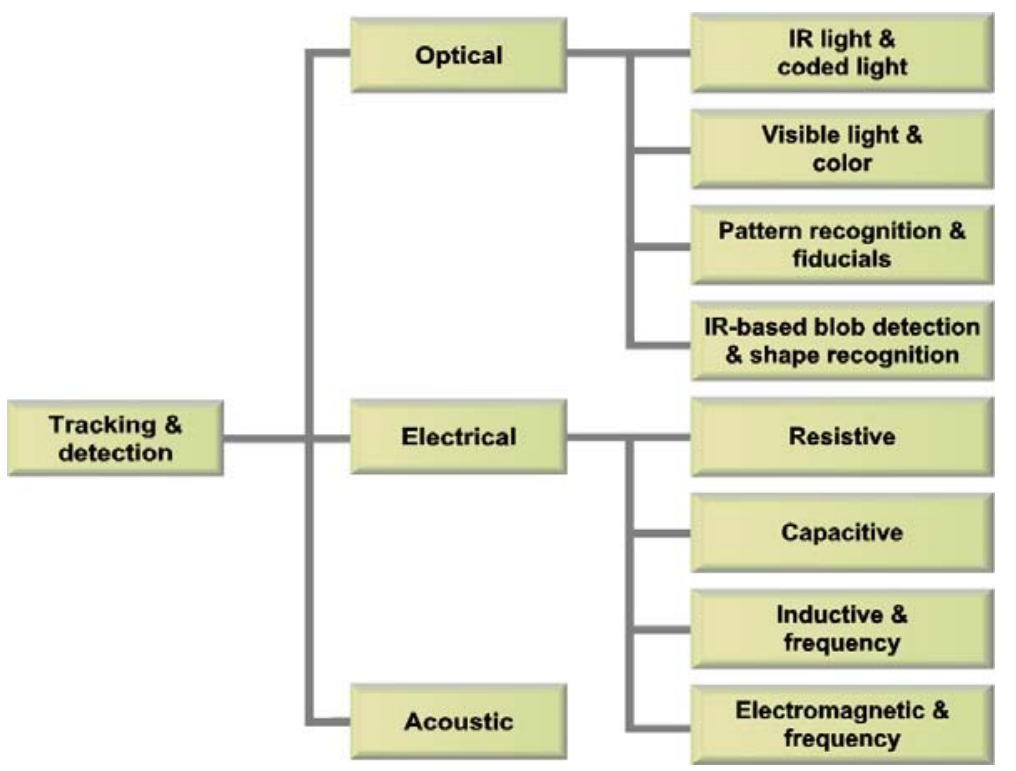

Figure 2. Types of tabletop tracking

Beside interaction and tracking, a third, more technical way of classifying tabletop systems exists. By taking the position of the individual technical components and other general aspects into account, such as multi-user capability, touch detection, TUI interaction, ID, and state (such as passive, idle, active, mode of use, etc.), a classification matrix (see Figure 3) can be established. 


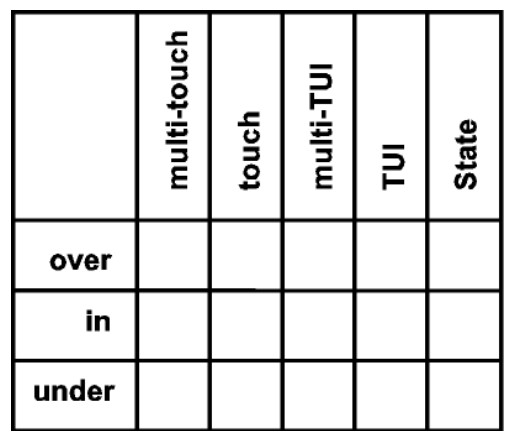

Figure 3. Classification matrix of tabletop systems

The above matrix template will be used in section 3 to give a quick overview of systems' capabilities along with a brief description of key research results in the past decade.

\section{Image Display}

In principle, two basic ways of displaying information exist: front- and backprojection. While front-projection refers to when the user and the image source are on the same side of the interaction plane, back-projection is when they are on different sides of the interaction plane. In both cases, projectors are used most frequently, but some back-projection systems already employ flat screens based on LC- or plasma technology. Figure 4 gives an overview of the employed technologies.

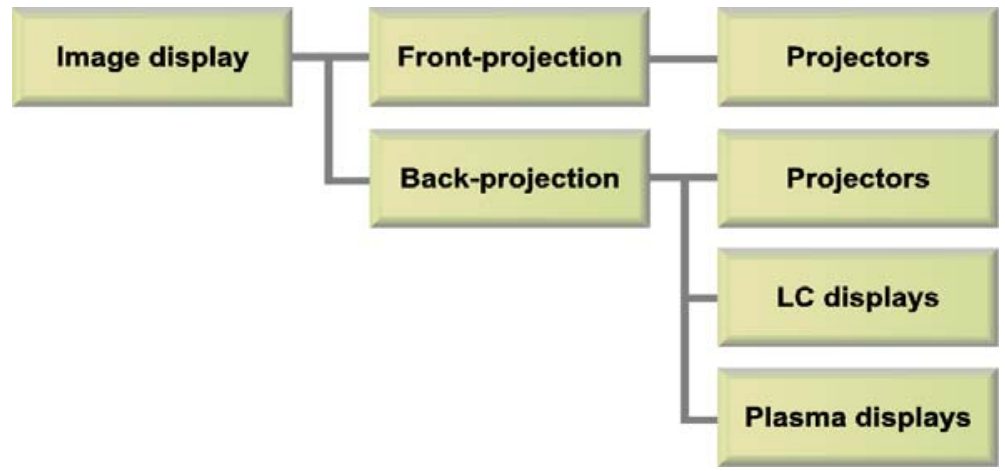

Figure 4. Display technologies for tabletop systems 


\section{Technical Challenges and Motivation}

Tabletop interaction strongly depends on the involved technology. Various kinds of tracking technologies, projection systems, and sophisticated interaction devices have been realized and combined into new groupware for collocated multi-user environments. Most of these systems suffer from technical imperfections either in tracking, identification, interaction, or image display. They also suffer from the fact that a light-path is required for the projection and the camera's image acquisition. Resolving these problems and realizing a table where a user can actually sit comfortably imposes requirements on the display as well as on all the other technical systems involved.

During the past decade tabletop research has often been focused on supporting collaborative teamwork, in particular brainstorming and creativity sessions. In such phases of teamwork, intuitive handling of the devices sitting on a table is crucial. Supporting team-oriented idea gathering through tabletop interaction implies that all participants are able to interact simultaneously. Even when simultaneous user input does not take place, the system should be able to detect and identify multiple objects on the surface. Here, tabletop systems must overcome the single user principle and the underlying idea of controlling the system by one single interaction device.

Realizing tabletop systems differs completely from implementing typical interaction behaviours on vertical interactive surfaces, where multiple commercial solutions already exist. While vertical interactive surfaces follow the so-called whiteboard metaphor, relying on a single-user presentation and single-device penbased input, the tabletop metaphor is inherently a multi-user system, triggered by intended and unintended input. For instance, simple writing, which is not typically problematic on a vertical whiteboard, turns out to be a delicate task on a horizontal tabletop. This is because tabletop users typically touch the interaction surface at multiple points with their hand and not only at the intended interaction point of the device. So, the system should be able to distinguish between intended and unintended input in order to generate correct results. This situation becomes even more complex if users consciously use their fingers in addition to devices to interact with the system. For this situation, systems must be able to combine tangible user interfaces with touch input on the same surface.

In order to meet the technical challenges mentioned above, continuous work on improving registration, display, and system technology for tabletop interaction is required. Another goal is to integrate all the technical components from above and below the tabletop into the tabletop itself, thus realizing a system that is more intuitive to handle and more ergonomic to work with. The following section presents milestones from the past decade on the way to such integrated systems. 


\section{Milestones on the Way to an Integrated System}

For about 20 years, tabletop interaction has inspired researchers all over the world. While the technology required was placed 'over the tabletop' in the early systems, recent research focuses on the integration of all components 'under the tabletop' or even 'in the tabletop'. The following overview presents some important milestones on the way to realizing an intuitive tabletop system.

\section{Digital Desk - 1991}

In 1991, Wellner [3] recognized that the electronic desktop on the screen is separated from the user's physical desk. He pointed out the basic motivation for doing tabletop research in general: electronic documents lack many properties of physical paper, while physical paper lacks many useful properties of electronic documents. With DigitalDesk, he created a system that allows interacting with digital (projected) paper with a bare finger. The system uses a front-projection as well as a front image acquisition of the user's hand and finger. In order to achieve a reasonable update rate, the camera's image was first configured with a low resolution when detecting the positions of the finger and hand. Once this was detected, a second high-resolution image with a close-up view could detect the characters of the underlying document. Using this optical tracking and recognition system, the underlying application was capable of reading numbers from a piece of paper and entering them into a digital calculator application (see Figure 5).
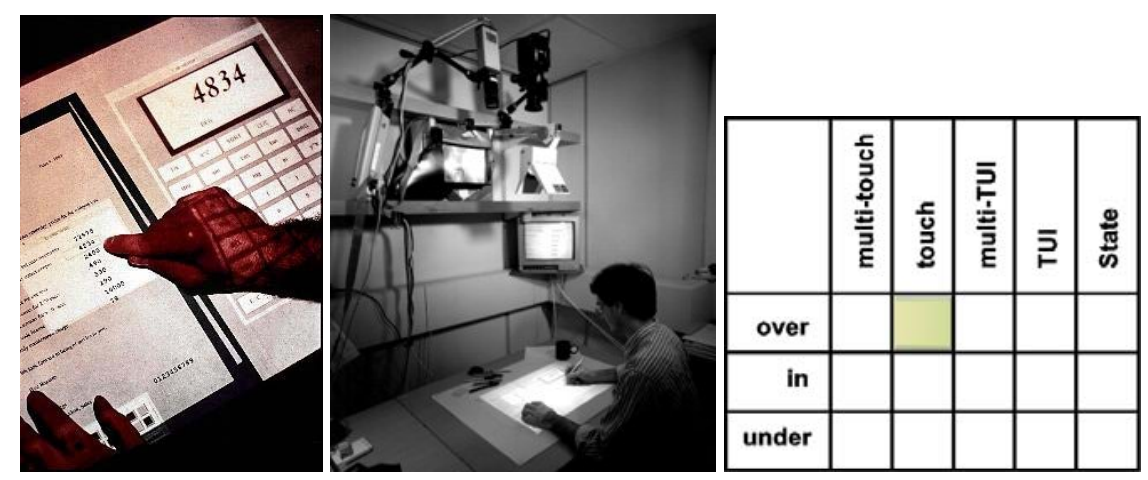

Figure 5. DigitalDesk Transferring content from analogue paper to a digital application [3]

Normal daylight and office lighting conditions caused problems. Also, a frame rate of 25 - 30 frames per second created irritating latencies. At this point, the system did not require any additional interfaces than the fingers. 


\section{ActiveDesk - 1995}

In 1995, Fitzmaurice et al. [4] introduced ActiveDesk, which allowed using interaction devices other than the fingers. Using a back-projected digitizing board, it was also possible to interact with a stylus. Fitzmaurice also introduced the term, graspable user interface, which is the physical representation of the graphical user interface, having the same abbreviation of GUI. This required the ability to use tools other than the stylus to interact with the system. The graspable user interface was dynamically associated to a virtual object if it was placed upon it. In order to release it, the user had to lift the GUI away from the surface. Finally, in order to retrieve information about the bricks' positions, an electromagnetic tracking system was used with its receiving antennae serving as a brick for interaction. The receivers were cable-bound and could mechanically interfere with each other. Thus, mainly bimanual interaction was supported, as shown in Figure 6.
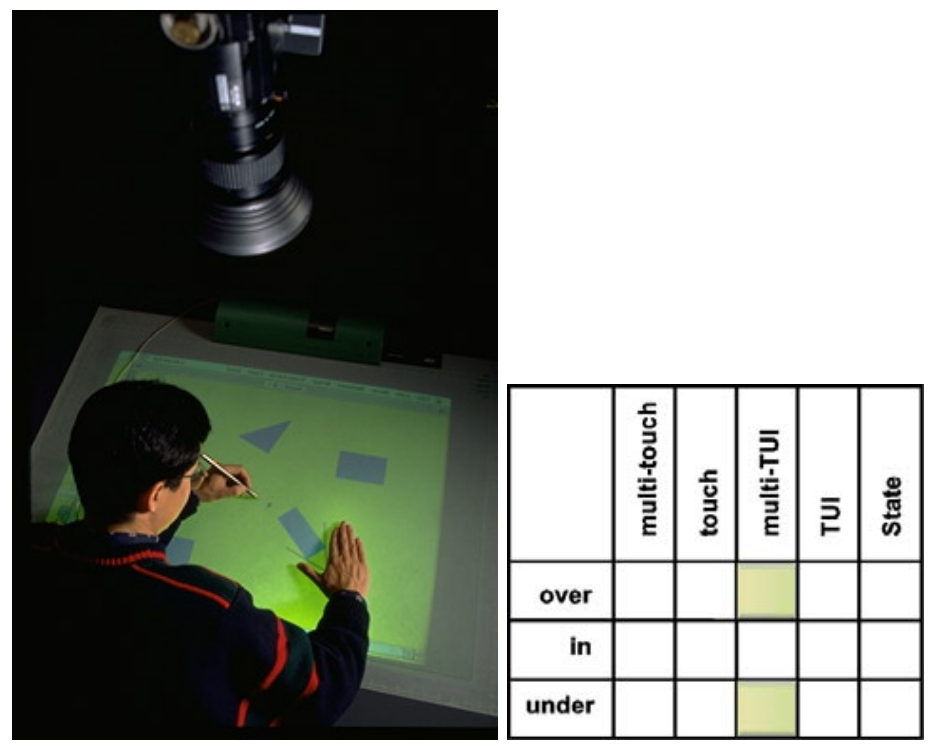

Figure 6. Bimanual interaction on ActiveDesk [4]

Unlike DigitalDesk, ActiveDesk uses a back-projection onto the tabletop. Thus, the tabletop system's active components are above the tabletop (tracking) as well as under the tabletop (projection). 


\section{The metaDESK - 1997}

In 1997, metaDESK was introduced by Ullmer et al. [5]. The metaDESK addressed the problem that interaction either with fingers only or with a dynamic assignment of interaction bricks to virtual objects did not allow sufficient interaction capabilities. Also, the interaction bricks were not intuitive to handle. Thus, Ullmer et al. introduced the term, Tangible User Interface (TUI). These interfaces disclose their inherent functionality and influencing the tabletop system by their shape. Employing shapes known to users from their daily life makes the handling of tabletop systems more intuitive (see Figure 7).
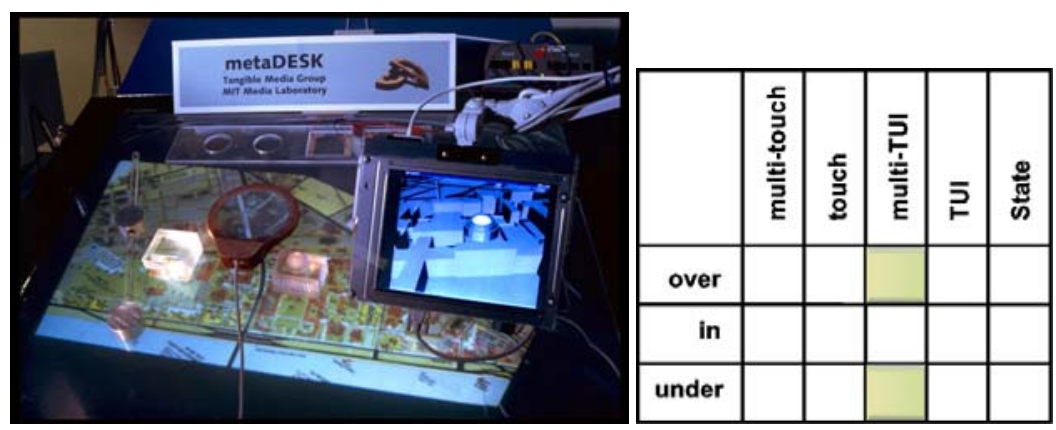

Figure 7. Typical TUI within metaDESK [5]

The metaDESK employs a vision-based tracking system using infrared (IR) light, which is back-projected onto the tabletop's surface together with the visible image. Since the objects will reflect the infrared light, they are visible to the camera, which is also mounted underneath the tabletop. In addition, an electromagnetic tracking system is used for the lens and the position of an additional LCscreen in order to provide a 3D-view of the scenery.

Again, to reinforce the fact, the system's components are above and below the tabletop.

\section{The BUILD-IT System - 1998}

The BUILD-IT system was introduced in 1998 by Fjeld et al. [6]. This system uses a front-projection onto a tabletop and a reflective IR tracking system. Applying computer-vision technologies to the acquired camera image allows multiple physical handles - the so-called bricks - to be detected on the table, which serves as a passive projection screen. Thanks to a combination of bricks that reflect IR light and a camera with an IR filter, the bricks are the only objects on the tabletop's surface that are visible to the camera. The bricks are dynamically associated 
to the virtual objects. Releasing the bricks is done by interrupting the free line-ofsight between the brick and the camera (see Figure 8).
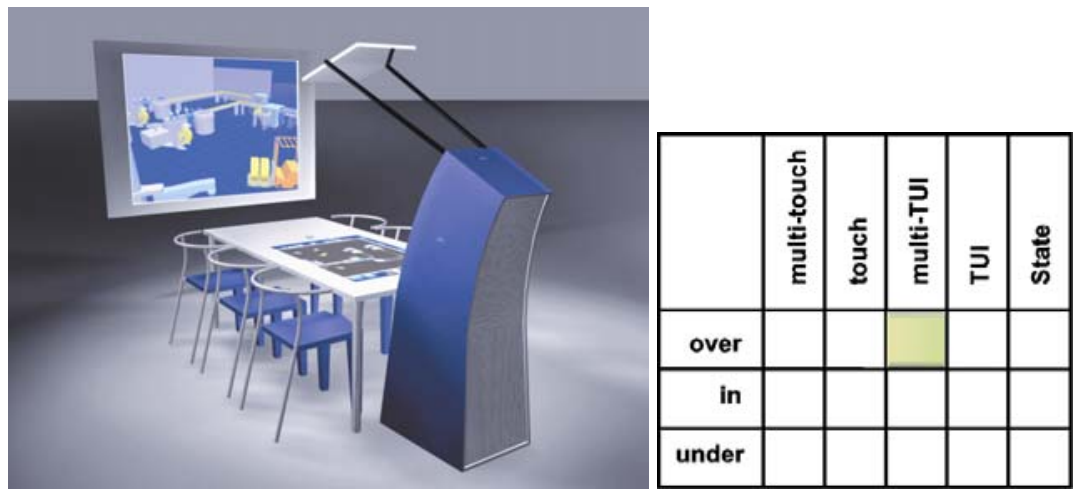

Figure 8. The BUILD-IT system [6]

Like metaDESK, the BUILD-IT system also offers a quasi-3D view on a vertical screen (see Figure 8), allowing a perspective view of a 3D scene.

For this system, all active components are located above the table, allowing for comfortable work in a sitting position.

\section{The Magic Table - 2003}

In 2003, Bérard [7] introduced the Magic Table, consisting of a regular whiteboard on which a user can write and sketch using regular ink. For special commands, such as copy, cut, or rotate, coloured tokens are used which are recognized by the camera above the table. Special gestures with the tokens are interpreted triggering another camera to perform a high-resolution scan of the sketches on the table. Next, a copy is projected over the original version, which can now be moved, rotated, or deleted (see Figure 9).
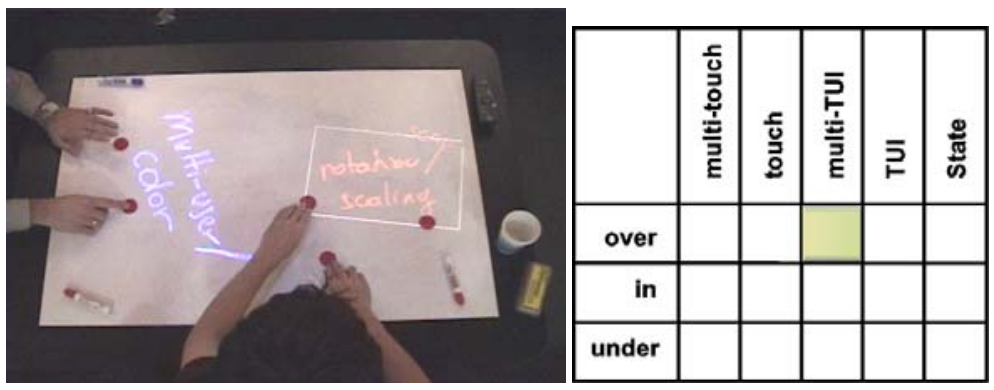

Figure 9. The Magic Table [7] 
The tabletop system's components are placed above the surface, requiring a free line-of-sight for the optical tracking.

\section{SenseTable $2000-2002$}

SenseTable was presented by Patten et al. [8] in 2001. The system uses two WACOM Intuos tablets, which allow inductive sensing. Since this kind of sensing is capable of tracking only two devices, the interaction devices were designed in such a way that the integrated coil is randomly switched on and off. This allows for a greater number of devices to be used, but results in a significantly lower update rate of about $1 \mathrm{~Hz}$. The so-called pucks were dynamically associated to a virtual object by placing the puck next to it. The object is released again by shaking the puck or removing it from the interactive surface.

The system uses a front-projection, and the information is also partly visible on the surface of the pucks. This allows clear identification of a puck's functionality, since this cannot be derived from its shape (see Figure 10).

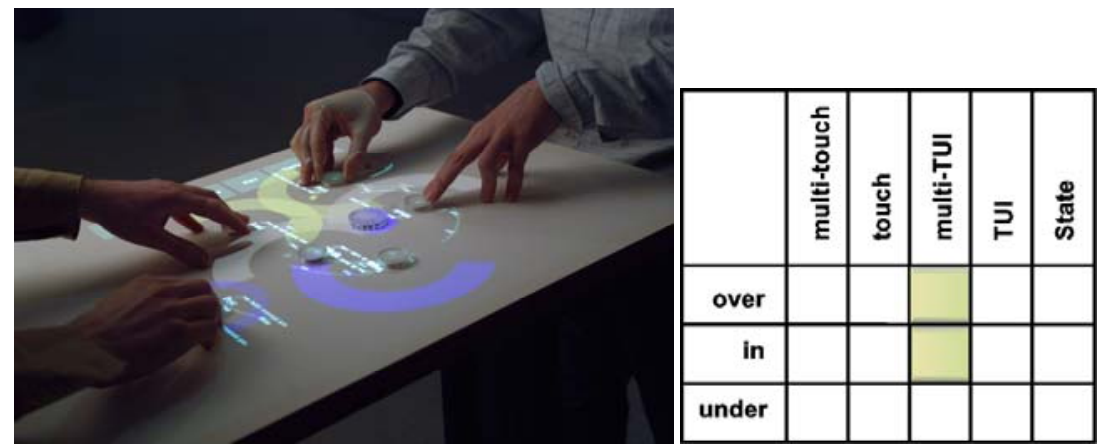

Figure 10. SenseTable with interactive puck [8]

SenseTable was also the first approach to integrate part of the tabletop system's technology into the tabletop, namely the tracking system. The system still uses a front-projection, which is partly camouflaged when the user interacts with the puck. Thus, the other part of the tabletop system's technology is still above the tabletop.

\section{DiamondTouch - 2001}

In 2001, DiamondTouch was introduced by Dietz et al. [9]. Much like SenseTable, this system uses a front-projection above the tabletop and has a tracking system 
that is integrated into the tabletop. However, the tracking system works electronically, i.e. a high-frequency signal is transmitted from the table through the user and is detected by receivers in each user's chair. Thus, the table can detect multiple touches from different users, while objects on the surface do not interfere with normal operation. When the user touches the table, a capacitive coupled circuit is closed. This circuit runs from the transmitter through the touch point on the table's surface, through the user to the user's receiver, and back to the transmitter (see Figure 11).

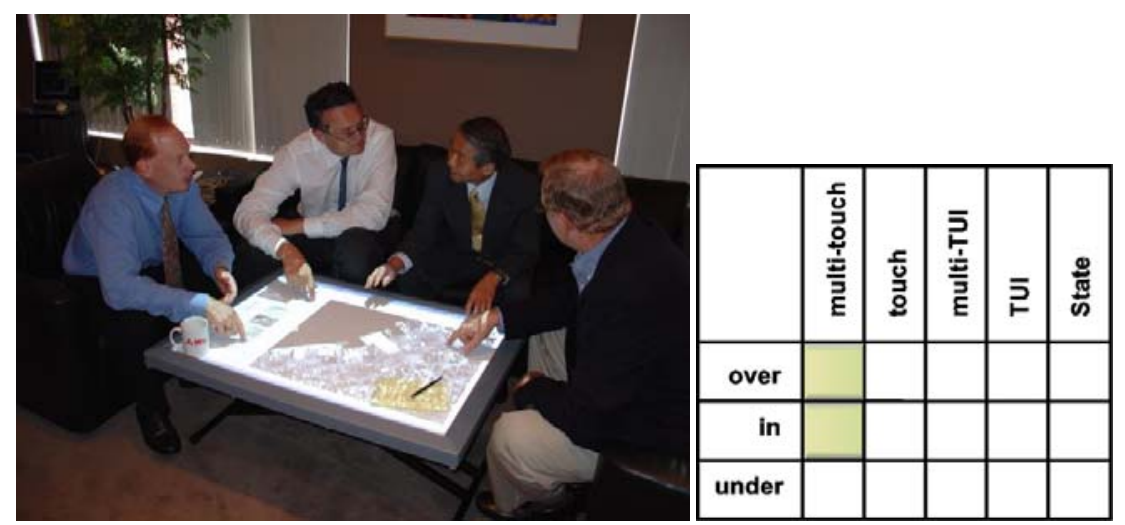

Figure 11. DiamondTouch [9]

Since the system is designed to detect multiple users' touches, no additional tools are available (TUI) for the intuitive operation of more complex functions.

\section{SmartSkin - 2002}

Rekimoto [10] introduced SmartSkin in 2002. Like DiamondTouch, it is a system that is able to detect multiple finger touches on a tabletop using capacitive sensing. In this case, the tracking system is again integrated into the tabletop, consisting of a mesh of transmitter and receiver electrodes. The resolution of the system also allows detecting gestures by interpreting the relative positions of detected blobs. Thus, gestures like grasping or zooming in/out can be detected by the system. Although the system cannot be influenced by most of the other objects on the table, there are some TUIs - so-called capacitance tags - available. However, as these tags are electrically grounded, they are not detected until the user touches them. Since the tags have a unique pattern, they can be unequivocally differentiated from normal finger touch patterns. 


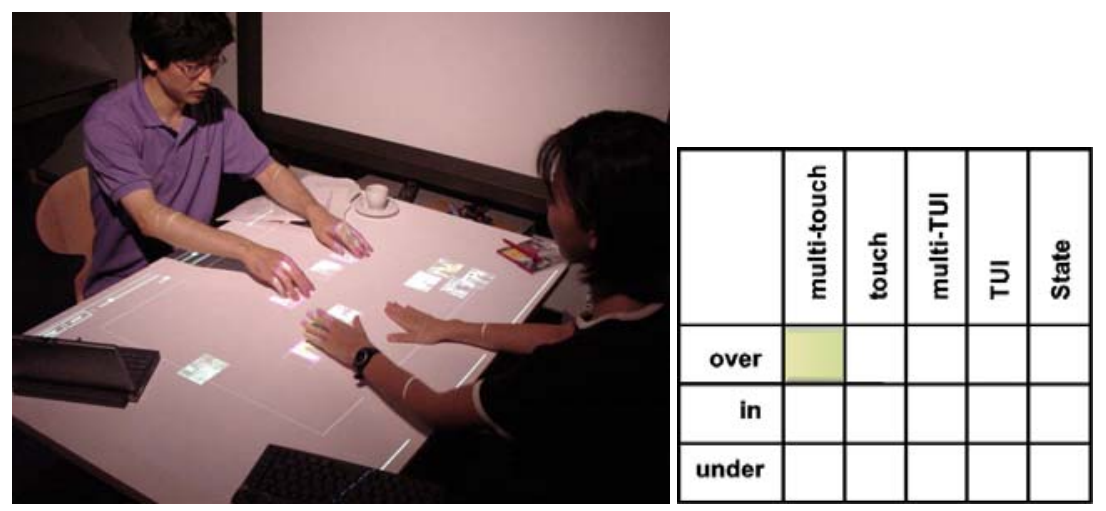

Figure 12. SmartSkin [10]

SmartSkin was one of the first systems that integrated both TUI and touch into the tabletop. In addition, the system was able to distinguish between a small number of TUIs by interpreting their shape. However, the system still uses a frontprojection and so, does not completely integrate all components into the tabletop.

\section{reacTable - 2003-2007}

The reacTable is a comparatively long project whose final system was presented by Jourdà et al. [11]. This tabletop system is capable of detecting TUIs and touch, but all of its components are located below the tabletop's surface. The system uses a back-projection as well as an optical tracking of fiducials from underneath the tabletop's surface. The TUIs are applied with fiducials that can be unequivocally detected by the camera. These also allow detecting orientation, which supports interaction capabilities. Since the tabletop is back-illuminated with IR light for detecting the fiducials, finger touch can also be easily detected. This allows modifying and adjusting preset parameters given by the TUIs.
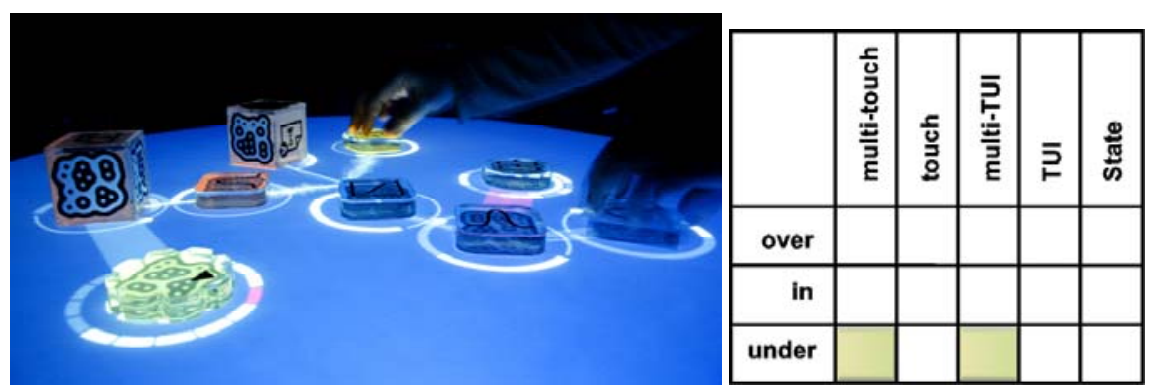

Figure 13. Interacting with TUIs on the reacTable [11] (courtesy to Xavier Sivecas) 
The TUIs are statically associated to a function (for a music application), but they do not show their inherent functionality intuitively by their shape. Thus, users need some training time in order to operate the system.

\section{PlayAnywhere - 2005}

In 2005, PlayAnywhere was introduced by Wilson [12]. It is a front-projected, computer-vision based interactive tabletop system. Both the wide-angle projection and the IR-based tracking system are located above the tabletop. The IR illumination is used to generate shadows, which can be seen by the camera (see Figure 14). Detecting the size of the shadow can determine not only a finger's position but also whether the finger touches the surface or not. Such detection is called hovering. So, PlayAnywhere is capable of taking the z-axis into account, i.e. it is possible to distinguish between pointing and touching.

If objects are applied with a visual code, they can also be detected by the system. Thus, it is also possible to integrate TUIs into the underlying application.
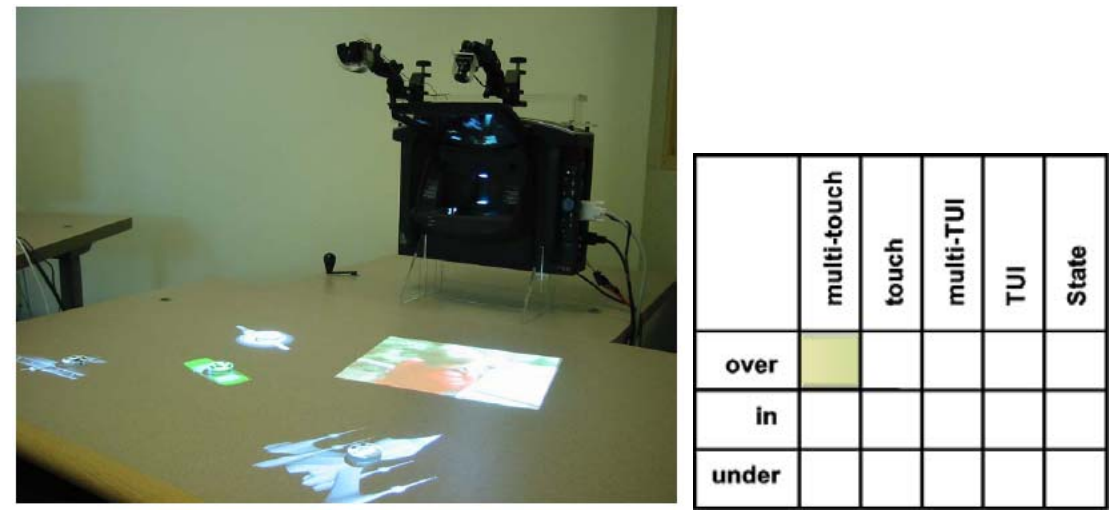

Figure 14. Working with PlayAnywhere [12]

\section{FTIR - 2005}

Multi-touch IR-based technology was introduced by Han [13][14] in 2005. The system uses a back-projection onto the tabletop's surface and an IR-based tracking system, which also requires a camera underneath the tabletop. Again, all components are below the tabletop. Unlike reacTable, the IT-light is not realized in a back-illumination but coupled into an acrylic overlay from the side. Within the acrylic, the IR light is completely internally reflected. At the position where a fin- 
ger is pressed onto the tabletop's surface, the total internal reflection is distorted and some IR light is coupled out. This amount of light can be seen by a camera, which is applied with a special IR pass-through filter (see Figure 15)
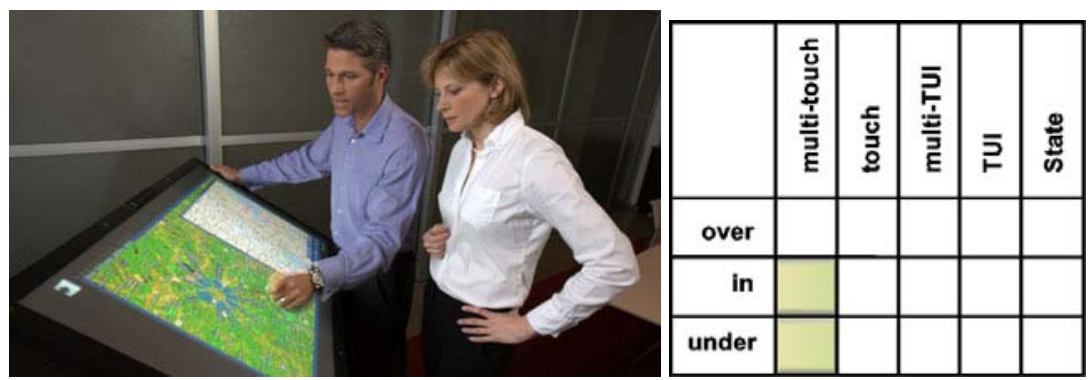

Figure 15. The FTIR system [13] [14]

The system was designed to support multi-touch, but due to the physical working principle, other objects on the tabletop's surface cannot be detected unless they are coated with a silicone layer, which is also able to couple out light from the acrylic tabletop. The camera image is used for blob detection, which again gives information about the fingers' positions.

\section{Ortholumen - 2007}

Ortholumen is a light pen based tabletop interaction system that can employ all the pen's spatial degrees of freedom (DOF). The pen's light is projected from above onto a horizontal translucent screen and tracked by a webcam sitting underneath, facing upwards. The system output is projected back onto the same screen (see Figure 16). The elliptic light spot cast by the pen informs the system of pen position, orientation, and direction. While this adds up to six DOFs, Piazza et al. [15] used up to four at a time.
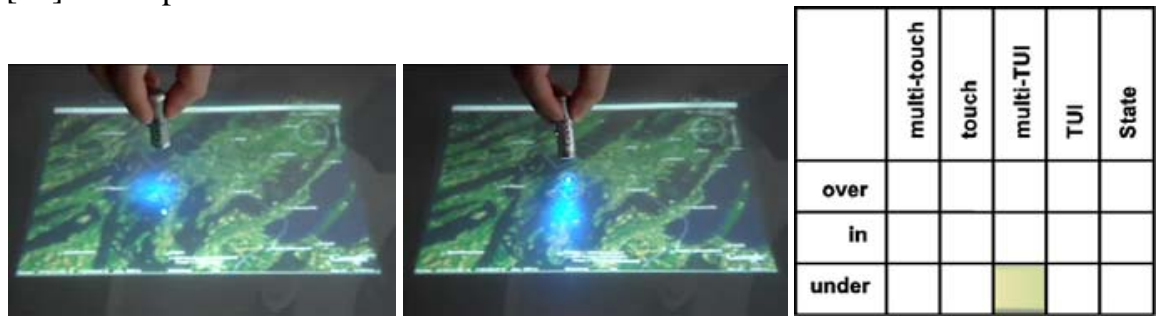

Figure 16. Ortholumen: Light-based navigation of Google Earth [15]

In order to better separate input and output light, they employed polarizing filters on the webcam and the projector lens. Two applications, painting and map naviga- 
tion, were presented. Ortholumen can be expanded to track multiple pens of the same or different colours. This enabled multi-pointer input, collaboration, and placed pens as external memory. Visible light, as opposed to infrared or radio, may be perceived more directly by users. Ortholumen employs only low-cost parts, making the system affordable to home users.

\section{InfracTables - 2005}

In 2005, Ganser et al. [16] proposed the InfracTables system. Within this system, all components are underneath the tabletop's surface; it uses a back-projection as well as an image acquisition from underneath. The devices are active, i.e. they are triggered by an IR synchronization-flash and respond with a device-specific bitcode. Based on this principle, several interaction devices were realized, such as stylus, eraser, ruler, calliper, ink dwell, etc. (see Figure 17).

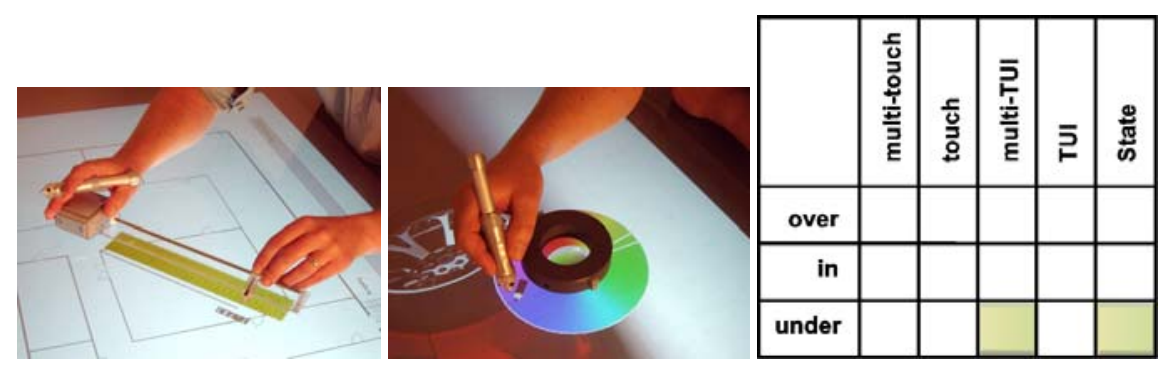

Figure 17. Working with InfracTables [16]

While a device's position on the tabletop can be determined by blob detection, ID and mode can only be determined by evaluating five subsequent camera frames. This means that the overall refresh frequency is divided by the number of bits being used for unequivocally determining the identity of each device. In the presented version, seven different devices with three states each could be detected.

\section{MightyTrace - 2008}

In 2008, MightyTrace was introduced by Hofer et al. [17]. This system focuses on integrating all technical components into the tabletop. The display as well as the tracking system is integrated into a single housing, and thus, it is possible to realize an ergonomic table that people can actually sit at. 


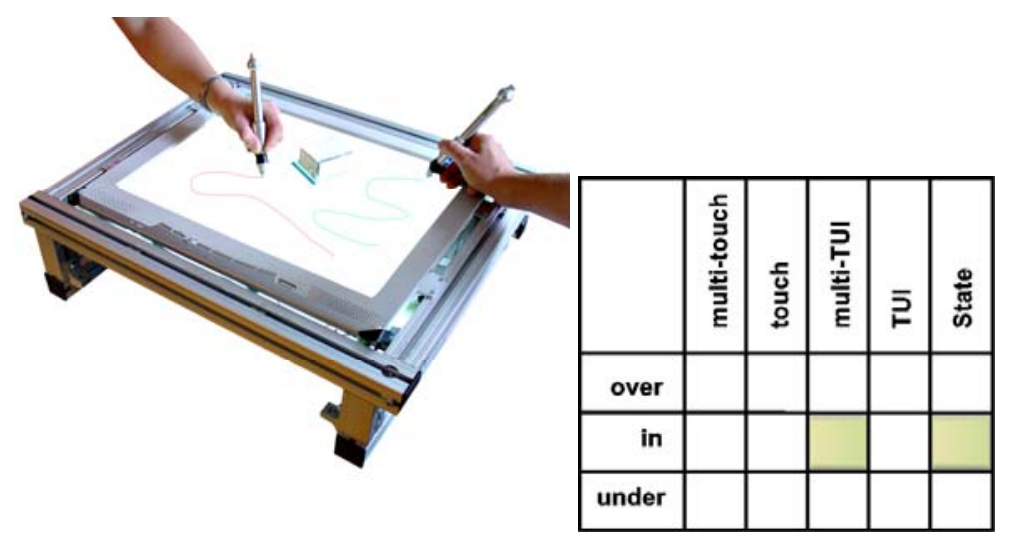

Figure 18: First prototype of MightyTrace [17]

Figure 18 shows an early prototype of MightyTrace, which employs IR sensors, being integrated into an LC-screen for tracking multiple devices on the tabletop. The tracking system consists of an array of IR-sensors that are placed behind the LC-matrix. The TUIs actively emit IR-light when being triggered by a synchronization flash. The emitted IR-light of the TUIs goes through all components of the LC-screen and is only marginally influenced by the content being displayed on the screen. The prototype reaches a resolution of $3 \mathrm{~mm}$ with an update rate of at least $100 \mathrm{~Hz}$. However, it only allows interaction using specialized active devices such as a stylus or interaction bricks.

\section{FLATIR - 2009}

An adaptation of the FTIR technology to an LC-screen was suggested by Hofer et al. [18]. This system, again, integrated all components into the tabletop. As in MightyTrace, IR-sensors behind the LC-matrix are used. In addition, the LCscreen is equipped with an FTIR-overlay, which allows multi-touch detection (see Figure 19). 


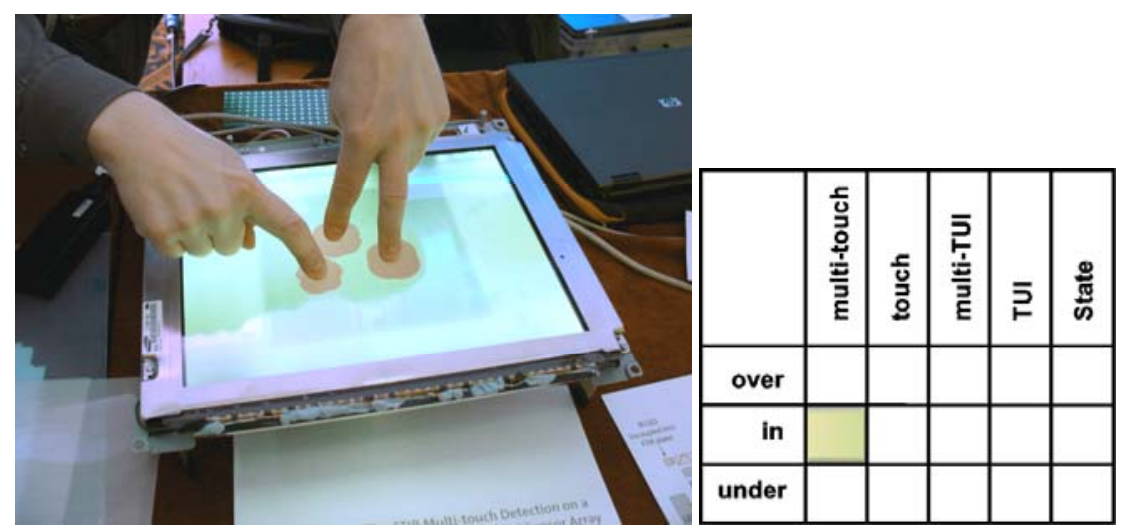

Figure 19: FLATIR: FTIR multitouch detection on an LC-screen [18]

Now, the blobs generated by the finger touches (visualized as red circles in Figure 19) can be analyzed and then used for an underlying application. However, it is no longer possible to distinguish between individual fingers and so finger touch can mainly be used for controlling a mouse pointer. After a dynamic association with a virtual object or functionality, the finger can also be used for performing and controlling more complex functions.

\section{Summary and Outlook}

This article classifies research activities within the field of tabletop interaction. In order to show the tendency of making the systems more intuitive to handle and integrating the technology completely into the tabletop, several of the most relevant research results were briefly introduced. While larger interactive spaces could only be realized with projection systems in the past, today's flat screen technology - in particular LC technology - offers interesting alternatives while becoming more and more affordable.

Using LC-technology tabletop systems become more ergonomic, since real tables can now be realized. So, it is now possible to actually sit at the table, because cameras and projectors are no longer in the way. Although this was possible by using systems with the technology 'above the tabletop', disturbing shadow casting that typically exists in front projection systems can now be avoided.

While current research into this new generation of tabletop systems supports either finger touch or TUI interaction, future research work such as TNT [19] will be able to integrate both, making tabletop systems even more intuitive to handle. In addition, future operating systems such as Windows 7 will support multiple mouse pointers, which will pave the way for new applications that benefit from multi-touch tabletop systems as described above. 


\section{References}

[1] Shen C, Ryall K, Forlines C, Esenther A, Vernier F, Everitt K, Wu M, Wigdor D, Morris M, Hancock M, Tse E (2009) Collaborative Tabletop Research and Evaluation: Interfaces and Interactions for Direct-Touch Horizontal Surfaces. In: Dillenbourg P, Huang J, Cherubini M (Eds.): Interactive Artifacts and Furniture Supporting Collaborative Work and Learning; Springer Science and Business Media, pp. 111-128, doi: 10.1007/978-0-387-77234-9_7

[2] Ishii H, Ullmer B (1997) Tangible Bits: Towards Seamless Interfaces between People, Bits and Atoms. In: CHI '97: Proceedings of CHI '97; ACM, New York, NY, USA, pp. 234241, doi: $10.1145 / 258549.258715$

[3] Wellner P (1991) The DigitalDesk Calculator: Tactile Manipulation on a Desktop Display. In: UIST '97: Proceedings of the ACM Symposium on User Interface Software and Technology (UIST '91); ACM, New York, NY, USA, pp. 27-33

[4] Fitzmaurice G. Ishii H, Buxton W (1995) Bricks: Laying the Foundation for Graspable User Interfaces. In: CHI '95: Proceedings of CHI 1995; ACM, New York, NY, USA, pp. 442449, doi: $10.1145 / 223904.223964$

[5] Ullmer B, Ishii H (1997) The metaDESK: Models and Prototypes for Tangible User Interfaces. In: Proceedings of UIST '97, ACM, New York, NY, USA, pp. 223-232, doi: 10.1145/263407.263551

[6] Fjeld M, Bichsel M, Rauterberg M (1998) BUILD-IT: An Intuitive Design Tool Based on Direct Object Manipulation. In: Wachsmut I, Fröhlich M (Eds.): Gesture and Sign Language in Human-Computer Interaction; Lecture Notes in Artificial Intelligence, Vol. 1371, Springer Berlin Heidelberg, pp. 297-308, doi: 10.1007/BFb0053008

[7] Bérard F (2003) The Magic Table: Computer-Vision Based Augmentation of a Whiteboard for Creative Meetings. In: Proceedings of IEEE International Conference in Computer Vision

[8] Patten J, Ishii H, Hines J, Pangaro G (2001) SenseTable: A Wireless Object Tracking Platform for Tangible User Interfaces. In: Proceedings of CHI 2001; ACM, New York, NY, USA, pp. 253-260, doi: 10.1145/365024.365112

[9] Dietz P, Leigh D (2001) DiamondTouch: A Multi-user Touch Technology. In: Proceedings of the $14^{\text {th }}$ Annual Symposium on User Interface Software and Technology UIST 2001, ACM, New York, NY, USA, pp. 219-226, doi: 10.1145/502348.502389

[10] Rekimoto J (2002) SmartSkin: An Infrastructure for Freehand Manipulation on Interactive Surfaces. In: Proceedings of CHI 2002; ACM, New York, NY, USA, pp. 113-120, doi: .org/10.1145/503376.503397

[11] Jourdà S, Geiger G, Alonso M, Kaltenbrunner M (2007) The reacTable: Exploring the Synergy between Live Music Performance and Tabletop Tangible Interfaces. In: Proceedings of the $1^{\text {st }}$ International Conference on Tangible and Embedded Interaction; ACM, New York, NY, USA, pp. 139-146, doi: 10.1145/1226969.1226998

[12] Wilson A (2005) PlayAnywhere: A Compact Interactive Tabletop Projection-Vision System. In: Proceedings of the $18^{\text {th }}$ Annual ACM Synposium on User Interface Software and Technology UIST 2005, ACM, New York, NY, USA, pp. 83-92, doi: $10.1145 / 1095034.1095047$

[13] http://cs.nyu.edu/ jhan/ftirtouch (accessed 12. March 2009)

[14] Han JY (2005) Low-cost Multi-touch Sensing through Frustrated Total Internal Reflection. In: Proceedings of UIST 2005; ACM, New York, NY, USA, pp. 115-118, doi: .org/10.1145/1095034.1095054

[15] Piazza,T, Fjeld M (2007) Ortholumen: Using Light for Direct Tabletop Input. In: Proceedings of IEEE TableTop 2007, IEEE Computer Society, Los Alamitos, CA, USA, pp. 193196, doi: 10.1109/TABLETOP.2007.23 
[16] Ganser C, Kennel T, Birkeland N, Kunz A (2005) Computer-supported Environment for Creativity Processes in Globally Distributed Teams. In: Proceedings of the International Conference on Engineering Design ICED 2005; pp. 109-110

[17] Hofer R, Kunz A, Kaplan P (2008) MightyTrace: Multiuser Tracking Technology on LCDisplays. In: Proceedings of CHI 2008; ACM, New York, NY, USA, pp. 215-218, doi: $10.1145 / 1357054.1357091$

[18] Hofer R, Naeff D, Kunz A (2009) FLATIR: FTIR Multi-touch Detection on a Discrete Distributed Sensor Array. In: Proceedings of the Third International Conference on Tangible and Embedded Interaction TEI '09, ACM, New York, NY, USA, pp. 317-322, doi: $10.1145 / 1517664.1517730$

[19] Hofer R, Kunz A (2009) TNT: Touch 'n' TUI on LC-Displays. In: Proceedings of the $8^{\text {th }}$ International Conference on Entertainment Computing ICEC ’09, Paris, France, pp. 222-227, doi: 10.1007/978-3-642-04052-8_24 